\title{
A multi observers approach when observability index is higher than the state dimension - a case study -
}

\author{
Ihab Haidar, Jean-Pierre Barbot and Alain Rapaport
}

\begin{abstract}
We address a family of observation problems that would classically require the construction of an embedding, by a different approach which consists in the design of several estimators in parallel. In principle, for a dynamics in dimension $n$ with a scalar output $y$, each estimator uses the knowledge of only $n-1$ derivatives of the output, and the further derivatives are used to discriminate at any time among the estimators. Estimators are built here by roots tracking technique. We illustrate our approach on the parameter estimation of a polynomial dynamics. The simulations show that the final estimation jumps from one estimator to another when passing through observability singularities, or when the parameter suddenly changes, preserving a good estimation error.
\end{abstract}

\section{INTRODUCTION}

Consider a dynamics in $\mathbb{R}^{2}$ of the form

$$
\begin{aligned}
\dot{x} & =P(x, c), \\
\dot{c} & =0,
\end{aligned}
$$

where $P$ is polynomial, along with the on-line observation

$$
y(t)=x(t) .
$$

The objective is to reconstruct the unknown parameter $c$ with the measurements $y(\cdot)$. The dynamics (1)-(2) can have several stable and unstable equilibrium, depending on the parameter $c$. However, we shall assume that the system is initialized such that the solution $x(\cdot)$ converges asymptotic to an equilibrium $x^{\star}(c)$. An illustrative example is given by the following dynamics

$$
\dot{x}=P(x, c):=x(c-x)\left((c-x)^{2}-\frac{13}{2}(c-x)+11\right),
$$

with $c>0$ and $x(0)=x_{0} \in(0, c)$. One can easily check that the roots of $P$ are $x=0$ and $x=c$ and that one has $P(x, c)>0$ for any $x \in(0, c)$, whatever is $c>0$. Therefore, any solution with $x_{0} \in(0, c)$ converge asymptotically to $x=c$. However, when one studies the differential observability of the system, one can observe that the value of $c$ cannot be uniquely determined without the knowledge of the three derivatives of $y$. Indeed, as $P$ is a third order polynomial in $c$, the injectivity of the map $(x, c) \mapsto\left(y, \dot{y}, \cdots, y^{(n-1)}\right)$ (where $y^{(j)}$ denotes the $j$-th time derivative of $y$ ) requires to take $n=4$.

When the observability index $n$ is higher than the dimension of the state space, one has to consider an immersion in higher dimension for the observer design (see for instance

Ihab Haidar and Jean-Pierre Barbot are with QUARTZ, ENSEA, CergyPontoise, France barboteensea.fr, haidardensea.fr, Alain Rapaport is with MISTEA, Univ Montpellier, INRA, France alain.rapaporteinra.fr the works [1], [2], [3], [6], [11], [13]). A systematic approach to obtain an observer in the original coordinates with an exponential convergence consists in 1) determining an embedding to write the dynamics in the canonical observer form [9] in higher dimension, 2) building an observer in this canonical form, and 3) expressing the estimation back in the original coordinates (see for instance [12]). However, this approach presents several difficulties in the construction of the embedding and the lipschitzian extension of the dynamics outside the set of its natural existence (cf. [4], [5]).

In this work, we propose another approach in the case of polynomial dynamics. Instead of a single observer in higher dimension, we propose a series of observers in the same dimension (each of them following the roots of a polynomial whose coefficients depends on the observation) and a test, based on higher derivative of the observation, which can discriminate between the observers the one that will give the right estimate.

For polynomials of small order ( 2 or 3 ), the roots can be determined analytically, but this is generally not possible for higher order. Here, we propose a roots tracking method to follow dynamically the roots over the time. The proposed method is also interesting for order 2 and 3 because it provides a continuous estimation of the roots (and acts then as a filter). This approach of parameter reconstruction somewhat generalizes the one proposed in [10] for a particular dynamics of batch reactors.

\section{OBSERVABILITY INDEX CHARACTERIZATION}

Let $\mathscr{D}$ be a compact subset of $\mathbb{R}^{2}$ positively invariant by the dynamics (1)-(2). A sufficient condition for the construction of observers for system (1)-(2) on $\mathscr{D}$ is that the map $(x, c) \mapsto(y, \dot{y})$ is injective on $\mathscr{D}$ (see, e.g. [14]). When this condition loses to be satisfied somewhere on the validity domain, the usual constructions of observers in the system's dimension is not possible. Nevertheless, one can check the possibility of overcoming this singularity problem by considering further derivatives of the output. Indeed, if

$$
z=\Phi_{n}(x, c)=\left[\begin{array}{l}
y \\
\dot{y} \\
\vdots \\
y^{(n-1)}
\end{array}\right]
$$

defines an injective immersion on $\mathscr{D}$, for some $n \geq 2$, the extension of system (1)-(2) to $\mathbb{R}^{n}$ is possible (see, e.g., [8], [12]). In this case, a constructive method allowing the construction of an exponential observer for (1)-(2) on $\mathscr{D}$ in its original coordinates is proposed in [12]. 
We recall, from [9], the definition of strong differential observability of (1)-(2) over $\mathscr{D}$ :

Definition 2.1: System (1)-(2) is said to be differentially observable of order $n$ over $\mathscr{D}$, if $\Phi_{n}$ is an injective mapping and to be strongly differentially observable, if $\Phi_{n}$ is an injective immersion.

Observe that the injectivity index of system (1)-(2), which is defined by the smallest positive integer $n$ such that the map $\Phi_{n}$ is injective, could be smaller than the degree of $P$ in the parameter $c$. For example, in the case when

$$
P(x, c)=x(c-x)^{5} \text {, }
$$

the injectivity index of system (1)-(2) is $n=2$. This injectivity index may also be not finite, as we can show in the case when

$$
P(x, c)=x c^{2}
$$

This motivates the following assumption.

Assumption 2.1: Let $p$ be the degree of $P$ in the parameter $c$. There exists an integer $2 \leq n \leq p+1$ such that the mapping $\Phi_{n}$ is injective on $\mathscr{D}$.

Thus, under Assumption 2.1, a necessary condition for the strong differential observability is that $\nabla \Phi_{n}$, the Jacobian matrix of $\Phi_{n}$, has a full rank over $\mathscr{D}$.

Let us introduce the following subsets of $\mathscr{D}$

$$
\mathscr{D}^{\star}=\{(x, c) \in \mathscr{D}: P(x, c)=0\}
$$

and

$$
\mathscr{S}_{n}=\left\{(x, c) \in \mathscr{D}: \frac{\partial P}{\partial c}=\frac{\partial P^{\prime}}{\partial c}=\cdots, \frac{\partial P^{(n-2)}}{\partial c}=0\right\},
$$

for $n \geq 2$.

Proposition 2.1: Suppose that Assumption 2.1 holds. If $\mathscr{S}_{n} \backslash\left(\mathscr{D}^{\star} \cap \mathscr{S}_{2}\right)=\emptyset$ then system (1)-(2) is strongly differentially observable of order $n$ over $\mathscr{D} \backslash\left(\mathscr{D}^{\star} \cap \mathscr{S}_{2}\right)$.

Proof: Let $(\bar{x}, \bar{c}) \in \mathscr{D}$. Observe that we have

$$
\frac{\partial}{\partial c} L_{P}^{i} h=\frac{\partial}{\partial c}\left(\frac{\partial L_{P}^{i-1} h}{\partial x}\right) P+\frac{\partial L_{P}^{i-1} h}{\partial x} \frac{\partial P}{\partial c}, \quad i \geq 2
$$

we distinguish three different cases:

- $(\bar{x}, \bar{c}) \in \mathscr{D}^{\star} \cap \mathscr{S}_{2}$ : in this case we have $\frac{\partial}{\partial c} L_{P}^{i} h(\bar{x}, \bar{c})=0$, for every $i \geq 2$. By consequence, the rank of $\nabla \Phi_{n}$ is at most equal to one at $(\bar{x}, \bar{c})$.

- $(\bar{x}, \bar{c}) \notin \mathscr{S}_{2}$ : in this case $\nabla \Phi_{n}$ is of full rank at $(\bar{x}, \bar{c})$.

- $(\bar{x}, \bar{c}) \notin \mathscr{D}^{\star}$ : if $\frac{\partial P}{\partial c}(\bar{x}, \bar{c}) \neq 0$ then $\nabla \Phi_{n}$, is of full rank at $(\bar{x}, \bar{c})$. Otherwise, if $\frac{\partial P}{\partial c}(\bar{x}, \bar{c})=0$, from (8) we obtain the following

$$
\frac{\partial}{\partial c} L_{P}^{2} h(\bar{x}, \bar{c})=\frac{\partial}{\partial x}\left(\frac{\partial h}{\partial x} \frac{\partial P}{\partial c}\right) P(\bar{x}, \bar{c})=\frac{\partial P^{\prime}}{\partial c}(\bar{x}, \bar{c}) P(\bar{x}, \bar{c}) .
$$

Using an induction reasoning, the proof that $\nabla \Phi_{n}$ is of full rank at $(\bar{x}, \bar{c})$ derives straightforwardly from the fact that $\mathscr{S}_{n} \backslash\left(\mathscr{D}^{\star} \cap \mathscr{S}_{2}\right)=\emptyset$.

According to Proposition 2.1, the map $\Phi_{n}$ cannot define an injective immersion on $\mathscr{D}^{\star} \cap \mathscr{S}_{2}$, for any $n \geq 2$. This motivates the following assumption.
Assumption 2.2: The forward solutions of system (1)-(2) do not converge asymptotically to $\mathscr{S}_{2}$.

Concluding this section, the construction of an exponential observer for (1)-(2) on $\mathscr{D} \backslash\left(\mathscr{D}^{\star} \cap \mathscr{S}_{2}\right)$ is possible. However, as mentioned in the introduction, this requires the construction of an embedding in higher dimension which present some technical difficulties (cf. [4], [5], [12]). In the next section, a different approach based on the design of several estimators in parallel is proposed.

\section{A MULTI-OBSERVERS APPROACH}

Let $s=c-x$, and let the polynomial $Q$ defined by $Q(y, s)=$ $P(y, s+y)$. The value of $c$ is equivalently determined through the knowledge of $s$. More precisely, we have $c=s(t)+y(t)$ at any $t \geq 0$. The solution $s$ of the following equation

$$
Q(y(t), s(t))=\dot{y}(t), \quad t \geq 0,
$$

is in general not uniquely determined. But, since $Q$ is polynomial in $s$ of degree $p$, there exist at most $p$ solutions sectors $s_{1}, \cdots, s_{p}$ such that

$$
F\left(z, s_{i}\right):=Q\left(z_{1}, s_{i}\right)-z_{2}=0, \quad \forall i=1, \cdots, p .
$$

Notice that the number of such solutions could depend on $z(t)$ (it depends on the number of real roots $s$ of the equation (9)). At any time $t$, there exists at least one solution sector $s_{i}$ such that $s_{i}(t)=s(t)$. The multi-observers approach that we propose consists in computing in parallel $p$ estimators $\hat{s}_{i}(t)$ of these roots, at any time $t$. If there are less than $p$ exact solutions of $F(z(t), s)=0$ at time $t$, then some estimators $\hat{s}_{i}(t)$ would be (temporarily) irrelevant. The way to determine these estimators will be addressed in the next section. The final task to provide a single estimation of $s(t)$ is to determine among the $p$ estimators $\hat{s}_{i}(t)$ which one is the right one. For this purpose, we use the further derivatives of $y$ and the injectivity of the map $\Phi_{p+1}$ to build the following test

$$
T(z, s):=\left[\begin{array}{c}
L_{Q}^{2} h(z, s)-z_{3} \\
\vdots \\
L_{Q}^{p} h(z, s)-z_{p+1}
\end{array}\right]=0
$$

from which we know that there exists only one solution among the (exact) solutions $s_{i}(t)$ at any time. In practice, we shall choose the estimation $\hat{s}(t)=\hat{s}_{i^{\star}(t)}(t)$ for which $\hat{s}_{i^{\star}(t)}(t)$ minimizes the norm of $T\left(z(t), \hat{s}_{i}(t)\right)$ among the estimators $\left\{\hat{s}_{i}(t)\right\}_{i=1 \cdots p}$.

Remark 3.1: From Assumption 2.1, $n-1$ estimators (or equivalently $n-1$ further derivatives of the output) are needed in order to estimate $s(t)$ at any $t \geq 0$. By consequence, the test given by equation (11) could be excessif when $n \leq p$. For example, in the case when $P$ is given by equation (4) (we have $n=2$ and $p=5$ ) the test (11) is even not needed. In order to simplify the presentation, the complet test (11) is considered in the rest of this paper. 


\section{A ROOTS TRACKING METHOD}

When the degree of $P$ in the parameter (or equivalently in $s$ ) is less than 4 , there exist exact analytical expressions of the roots of $F(z, \cdot)$. For higher degrees, we propose to use a roots tracking method to determine the $p$ estimators $\hat{s}_{i}(\cdot)$, as solutions of a dynamical system. Indeed, even for lower degrees, the roots tracking method presents some advantages, the estimations $\hat{s}_{i}(\cdot)$ being provided as continuous functions of the time.

For each time $t \geq 0$, we consider the polynomial

$$
s \mapsto F(z(t), s),
$$

where $F$ is defined by equation (10). Let us denote the shift $p \times p$ matrix

$$
D=\left[\begin{array}{llll}
0 & 1 & & \\
& & \ddots & \\
& & & 1 \\
0 & & & 0
\end{array}\right]
$$

and define the singularity set

$$
\mathscr{S}:=\left\{(z, s) \in \mathbb{R}^{p} \times \mathbb{R}, \partial_{s} F(z, s)=0\right\},
$$

with the vector fields defined outside this set:

$$
\begin{aligned}
& G(z, s)=-\frac{\partial_{z} F(z, s) D z}{\partial_{s} F(z, s)} \\
& G_{K}(z, s)=G(z, s)-K \frac{F(z, s)}{\partial_{s} F(z, s)}, \quad(z, s) \in \mathbb{R}^{p} \times \mathbb{R} \backslash \mathscr{S},
\end{aligned}
$$

where $K$ is a positive parameter. Let

$$
\mathscr{M}:=\{t \geq 0, s \mapsto F(z(t), s) \text { has multiple roots }\} .
$$

In addition to Assumptions 2.2, we add the following one.

Assumption 4.1: The set $\mathscr{M}$ is of null measure i.e. $\mathscr{M}$ is composed of isolated times of singularity.

Assumption 4.1 supposes that the solutions of (10) crossing the singularity set $\mathscr{S}$ do not say in it. This is needed for the well-posedness of our roots tracking method.

At each time $t$, we consider the roots $s_{i}(t)$ of $s \mapsto F(z(t), s)$ of multiplicity one. We consider a root numbering such that

1) $s_{i}(\cdot)$ is defined on a union of time intervals of positive measure, denoted $I_{i}$

2) for any $t \in I_{i} \cap I_{j}$ with $i>j$, one has $s_{i}(t)>s_{j}(t)$.

Then, as long as $t$ belongs to $I_{i}$, one has $\partial_{s} F\left(z(t), s_{i}(t)\right) \neq 0$, and the dynamics of $s_{i}$ is well defined:

$$
\dot{s}_{i}(t)=G\left(z(t), s_{i}(t)\right) .
$$

Notice that as $F\left(z(t), s_{i}(t)\right)=0$ at any $t \in I_{i}$ then $s_{i}$ is also solution of the dynamics

$$
\dot{s}_{i}(t)=G_{K}\left(z(t), s_{i}(t)\right)
$$

for any $K>0$. Consider this later dynamics for $\hat{s}_{i}$ which is not an exact root:

$$
\dot{\hat{s}}_{i}(t)=G_{K}\left(z(t), \hat{s}_{i}(t)\right), \quad t \in I_{i} \text { such that }\left(z(t), \hat{s}_{i}(t)\right) \notin \mathscr{S}
$$

and the function

$$
V(z, s)=\frac{1}{2} F(z, s)^{2} .
$$

One can straightforwardly check that one has

$\frac{d}{d t} V\left(z(t), \hat{s}_{i}(t)\right)=-2 K V\left(z(t), \hat{s}_{i}(t)\right), \quad t$ s.t. $\left(z(t), \hat{s}_{i}(t)\right) \notin \mathscr{S}$.

Consider now that at time $t_{0} \in I_{i}, \hat{s}_{i}\left(t_{0}\right)$ is such that

$$
\begin{aligned}
& \left(z\left(t_{0}\right), \hat{s}_{i}\left(t_{0}\right)\right) \notin \mathscr{S} \text { and } \\
& \quad\left|\hat{s}_{i}\left(t_{0}\right)-s_{i}\left(t_{0}\right)\right|<\left|\hat{s}_{i}\left(t_{0}\right)-s_{j}\left(t_{0}\right)\right|, \quad \forall j \text { s.t. } t_{0} \in I_{j} .
\end{aligned}
$$

Then, for an arbitrary large value of $K$, the solution $\hat{s}_{i}(\cdot)$ will be arbitrarily closed to $s_{i}(\cdot)$ in an arbitrary short of time, and remains arbitrarily close to $s_{i}(\cdot)$ as long as $t \in I_{i}$ with $\left(z(t), \hat{s}_{i}(t)\right) \notin \mathscr{S}$.

We propose now to approximate the dynamics $\dot{\hat{s}}_{i}=$ $G_{K}\left(z(t), \hat{s}_{i}\right)$ in the neighborhood of the singularity set $\mathscr{S}$ such that it is well defined for any $t \geq 0$ and any $i \in\{1, \cdots, p\}$. For this, we consider the following vector field:

$$
\widetilde{G}_{K, M}(z, s)=-\frac{\partial_{z} F(z, s) D z}{\operatorname{sat}_{M}\left(\partial_{s} F(z, s)\right)}-K \frac{F(z, s)}{\operatorname{sat}_{M}\left(\partial_{s} F(z, s)\right)},
$$

where the $\mathrm{sat}_{M}$ function is defined as follows

$$
\operatorname{sat}_{M}(\xi)=\min (\max (\xi,-M), M)
$$

with $M$ a positive real number. Finally, to ensure that the solutions $\hat{s}_{i}, i \in\{1, \cdots, p\}$ leave the cone

$$
\mathscr{P}=\left\{\left(s_{1}, \cdots, s_{p}\right) \in \mathbb{R}^{p}, s_{1}>s_{2}>\cdots>s_{p}\right\}
$$

invariant in the neighborhood of the singularity, we make another approximation coupling the vector fields as follows

$$
\begin{aligned}
& \widetilde{G}_{K, M, \alpha, \beta, i}\left(z, s_{1}, \cdots, s_{p}\right)= \\
& \quad \widetilde{G}_{K, M}\left(z, s_{i}\right)\left(1-\sigma_{i} \alpha e^{-\beta\left|s_{i-}-s_{i}\right|}\right)\left(1+\sigma_{i} \alpha e^{-\beta\left|s_{i}-s_{i+}\right|}\right)
\end{aligned}
$$

where $\sigma_{i}=\operatorname{sign}\left(\widetilde{G}_{K, M},\left(z, s_{i}\right)\right)$ and

$$
s_{i-}=\left\{\begin{array}{cc}
s_{i-1}, & i>1 \\
+\infty, & i=1
\end{array} \quad s_{i+}=\left\{\begin{array}{cc}
s_{i+1}, & i<p \\
-\infty, & i=p
\end{array}\right.\right.
$$

where $\alpha$ and $\beta$ are positive parameters. Notice that choosing large values of $\beta$ implies that the correcting multiplicative terms modify the original dynamics only in small neighborhoods of multiple roots, which according to Assumption 4.1 occurs only at isolated times, while the parameter $\alpha$ determines the amplitude of this dynamics perturbation. Observe also that the sign functions $\sigma_{i}$ are in such a way that solutions of

$$
\dot{\hat{s}_{i}}=\widetilde{G}_{K, M, \alpha, \beta, i}\left(z(t), \hat{s}_{1}, \cdots, \hat{s}_{p}\right), \quad i=1, \cdots, p,
$$

estimating different roots of (10) are pushing between each other in the neighborhood of singularities in order to keep $\mathscr{P}$ invariant.

We show in simulations that the solution of the dynamics (12) with initial condition in $\mathscr{P}$ can follow the exact roots $s_{i}(\cdot)$ over the time, provided it is well initialized. This method implies that the estimators $\hat{s}_{i}$ are initialized such that 

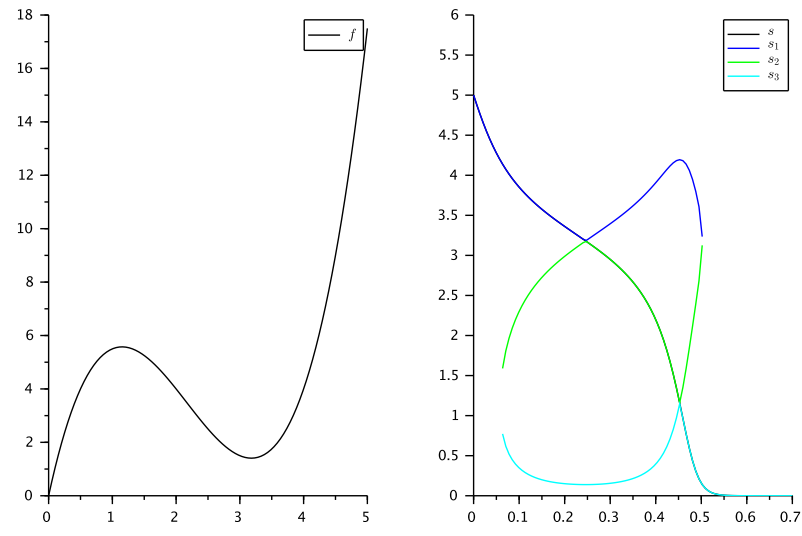

Fig. 1. Left: the function $f$. Right: the exact solutions of equation (14).

after a short period of time (due to the fast convergence of the function $V$ to 0 ), one of them has a norm of the test vector $T(\cdot)$ sufficiently small, at almost any future time. However, at initial time, one has no information at all about the location of the roots $s_{i}$. Either, some a priori information is available or one has to reinitialize, regularly on a short period of time, theses estimators in the very first initial stage. Such situation could also occur when the number of real roots increase. This last point will be discussed in further detail in a future work.

\section{EXAMPLE}

Let us consider the following dynamics in $\mathbb{R}^{2}$

$$
\left\{\begin{aligned}
\dot{s} & =-x f(s) \\
\dot{x} & =x f(s)
\end{aligned}\right.
$$

where $f: \mathbb{R} \rightarrow \mathbb{R}$ is given by

$$
f(s)=s\left(s^{2}-6.5 s+11\right),
$$

along with the online observation

$$
y=x \text {. }
$$

Observe that $\dot{s}(t)+\dot{x}(t)=0, \forall t \geq 0$. Consequently, we have

$$
s(t)+x(t)=s(0)+x(0), \quad \forall t \geq 0 .
$$

Let $c=s(0)+x(0)$. The analysis of system (13) can then be equivalently reduced to that of system (1)-(2) where, in this case, the polynomial function $P$ is given by

$$
P(x, c)=x f(c-x) .
$$

As presented in the introduction, we shall consider the compact positively invariant domain

$$
\mathscr{D}=\left\{(x, c) \in \mathbb{R}^{2}, 0 \leq x \leq c\right\} .
$$

In the case of this system, we have

$$
\mathscr{D}^{\star}=\{(x, c) \in \mathscr{D}: x=0 \text { or } x=c\} .
$$
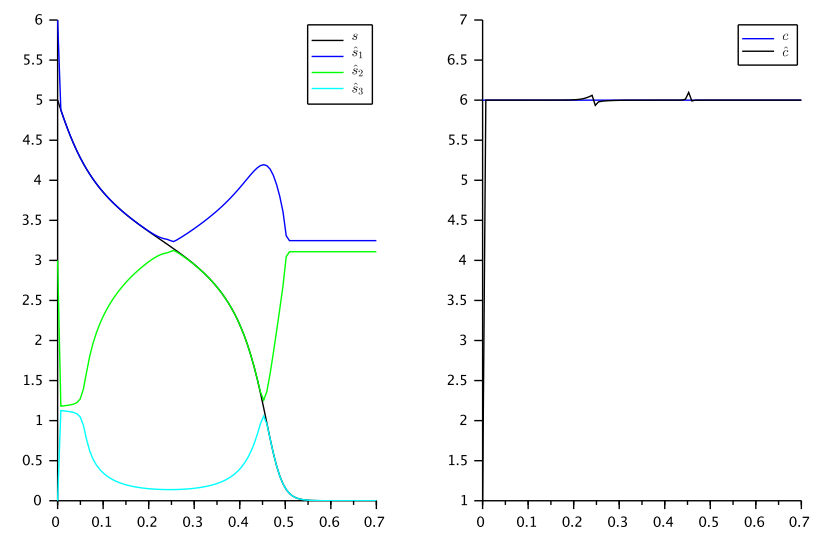

Fig. 2. Left: the estimated solutions of equation (14). Right: the constructed parameter $\hat{c}$ superposed with the exact parameter $c$.

In order to verify the conditions in the statement of Proposition 2.1, let us first calculate the derivative of $P$ with respect to $x$

$$
P^{\prime}(x, c)=f(c-x)-x f^{\prime}(c-x)
$$

and the derivative of $P$ and $P^{\prime}$ with respect to $c$

$$
\frac{\partial P}{\partial c}=x f^{\prime}(c-x), \quad \frac{\partial P^{\prime}}{\partial c}=f^{\prime}(c-x)-x f^{\prime \prime}(c-x) .
$$

One can easily verify, with the expression of the function $f$, that $\mathscr{S}_{3} \backslash\{0\}=\emptyset$. Knowing that $\Phi_{4}$ is injective over $\mathscr{D}$ then, thanks to Proposition 2.1, system (13) is strongly differentially observable of order 4 over $\mathscr{D} \backslash\{0\}$. Observe also that $x=0$ is a repulsive equilibrium point in the case of system (13), which is in conformity with Assumption 2.2.

In this example, following Section III, we have that the manifold

$$
F(z, s)=z_{1} f(s)-z_{2}=0
$$

is composed by three solutions sectors over which the test procedure is given by

$T(z, s)=\left[\begin{array}{c}z_{2} f(s)-z_{1} z_{2} f^{\prime}(s)-z_{3} \\ z_{3} f(s)-\left(2 z_{2}^{2}+z_{1} z_{3}\right) f^{\prime}(s)+z_{1} z_{2}^{2} f^{\prime \prime}(s)-z_{4}\end{array}\right]=0$.

In practice we choose at time $t$ the estimator $\hat{s}_{i}(t)$ that gives the minimal value of $\left|T_{1}\left(z(t), \hat{s}_{i}\right)\right|$. Indeed, we do not consider $T_{2}\left(z(t), \hat{s}_{i}\right)$ because the set of times $t$ for which $\left\|T\left(z(t), s_{i}(t)\right)\right\|=0$ is of null measure. Therefore we do not need to use $z_{4}$, the third derivative of the output.

To show the effectiveness of our method to reconstruct the parameter $c$ corresponding to system (13), we have chosen $x_{0}=1$ and $c=6$. The parameters $K, M, \alpha_{i}$ and $\beta_{i}$ relative to the estimators (12) have been chosen as follows

$$
K=100, M=100, \alpha_{i}=2, \beta_{i}=5, \quad i=1,2,3 .
$$

The initial conditions of the estimators have been chosen as $\hat{s}_{1}(0)=6, \hat{s}_{2}(0)=3, \hat{s}_{3}(0)=0$. The simulations was 

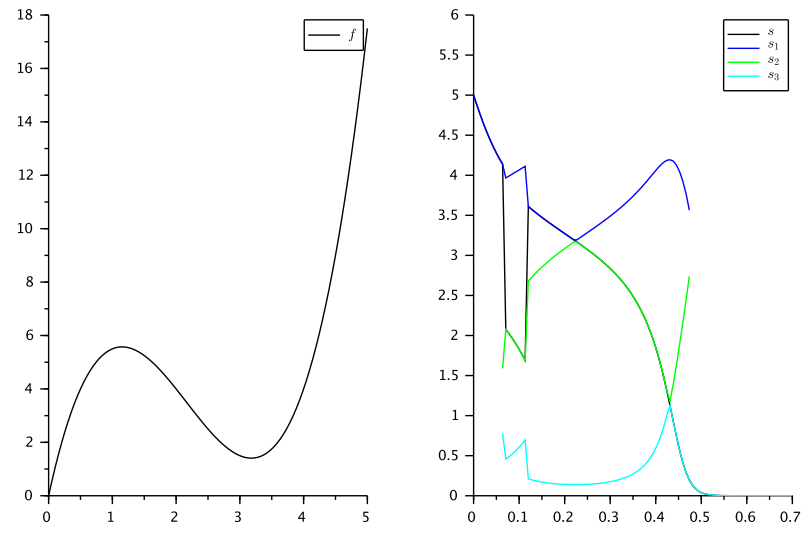

Fig. 3. Left: the function $f$. Right: the exact solutions of equation (14) with piecewise constant $c$.

performed with Scilab software with the classical Euler scheme and a dicretization step equal to $h=10^{-5}$.

Assuming the perfect knowledge of $z(t)$ at any time $t$, that is the perfect knowledge of the derivative of the output $y(\cdot)$, we can reconstruct the parameter $c$. On Figure 1-right we show the exact roots $s_{1}, s_{2}$ and $s_{3}$ solutions of (14) together with $s$ the solution of system (13). On Figure 2-left we show the estimated solutions $\hat{s}_{1}, \hat{s}_{2}$ and $\hat{s}_{3}$ together with $s$. Figure 2right shows the performance of our method to construct the parameter $c$. Let us underline that the number of the exact roots (corresponding to real roots) is changing with time: one, three and one again (see Figure 1-right), while our method always determine three estimators, but only a valid one is selected by the test function, at almost any time. One can observe on Figure 2 that the unknown parameter $c$ has been reconstructed before passing the singularity. To test the robustness of our method, we have simulated sudden changes of the parameter $c$, as a piecewise constant function:

$$
c(t)=\left\{\begin{array}{cc}
4 & t \in\left[t_{0}, t_{1}\right] \\
6 & \text { elsewhere }
\end{array}\right.
$$

with $t_{0}=0.07, t_{1}=0.12$ (with the same conditions of the previous simulations), so that the estimator has to change the choice of $\hat{s}_{i}$ over time, which illustrates the interest of our method that consists in computing estimations of all the roots at any time. Figure 3-left shows the estimated solutions $\hat{s}_{1}, \hat{s}_{2}$ and $\hat{s}_{3}$ corresponding to equation (14) together with $s$. Figure 4-right shows the performance of our method to construct the piecewise constant parameter given by (15).

Here, we have assumed the perfect knowledge of the vector $z(t)$ at any time $t$, which is hardly accessible in practice. We have then simulated our observer with $\hat{z}(\cdot)$ given by a numerical differentiate instead of $z(\cdot)$. In the initial stage, we have also considered a short delay before computing the estimators $\hat{s}_{i}(\cdot)$, for the differentiator $\hat{z}(\cdot)$ to converge. A simple high-gain differentiator is considered in
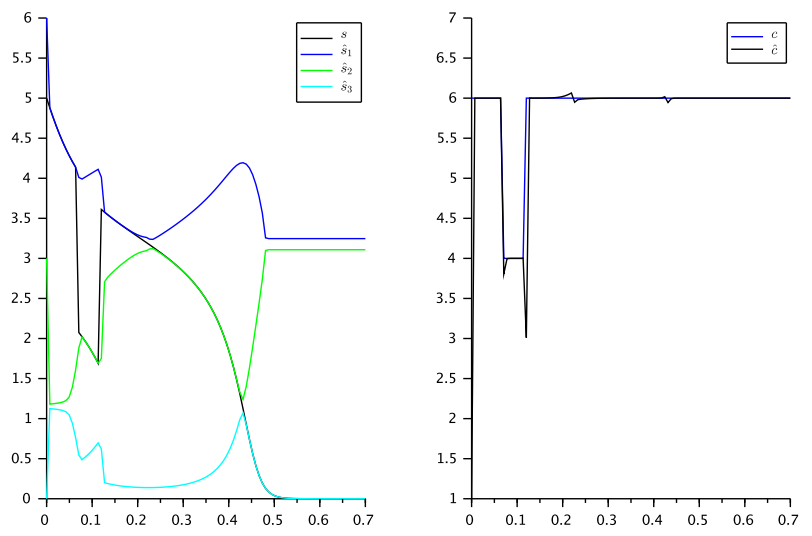

Fig. 4. Left: the estimated solutions of equation (14), with piecewise constant parameter $c$. Right: the constructed parameter $\hat{c}$ superposed with the exact parameter $c$.

order to compute $z_{2}$ and $z_{3}$

$$
\begin{aligned}
& \dot{\hat{z}}_{1}=\hat{z}_{2}-\frac{k_{1}}{\varepsilon}\left(\hat{z}_{1}-y\right) \\
& \dot{\hat{z}}_{2}=\hat{z}_{3}-\frac{k_{2}}{\varepsilon^{2}}\left(\hat{z}_{1}-y\right) \\
& \dot{\hat{z}}_{3}=-\frac{k_{3}}{\varepsilon^{3}}\left(\hat{z}_{1}-y\right)
\end{aligned}
$$

with $\varepsilon=10^{-3}, k_{1}=3, k_{2}=3, k_{3}=1$. On Figure 7 we depict the output and its first two derivatives together with the estimated ones given by system (16). Similarly, as in the case of exact derivatives of the output, we depict on Figures 5 and 6 the performance of our method to reconstruct the parameter $c$ with estimated output's derivatives.

\section{CONCLUSION}

In this paper we deal with the problem of parameter reconstruction when observability index is higher than the state dimension. We treat the case of planar polynomial dynamics and give an alternative approach compared to what exist in the literature, based on multiple observers. The parameter reconstruction problem we have studied is equivalent to an observer design problem for systems that are observable but with singularities of the observability map. Our method can be easily extended to the cases for which $P(x, c)$ is a rational fraction without pole in the invariant considered domain. This could be the matter of a future work.

\section{ACKNOWLEDGMENTS}

Authors would like to thank ENSEA for BQR funding helping to the preparation of this work. This work was also supported by the LabEx NUMEV incorporated into the I-Site MUSE. 

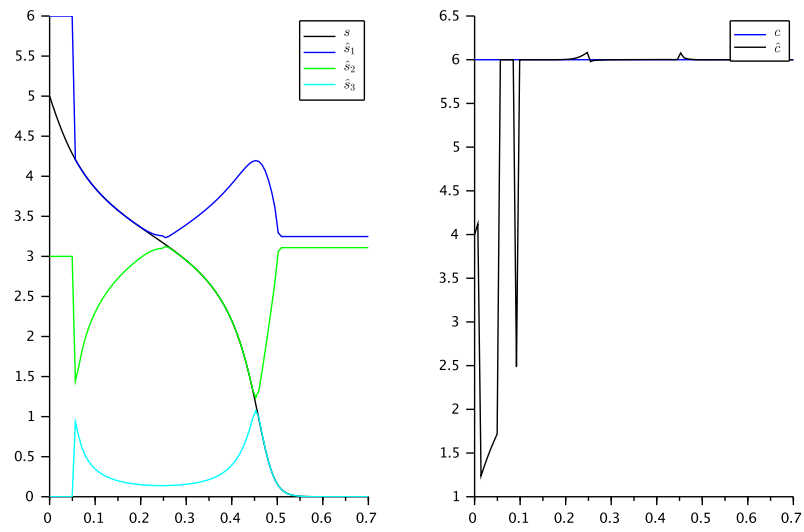

Fig. 5. Left: the estimated solutions of equation (14), with constant parameter $c$ in the case of estimated output's derivatives. Right: the constructed parameter $\hat{c}$ superposed with the exact parameter $c$.
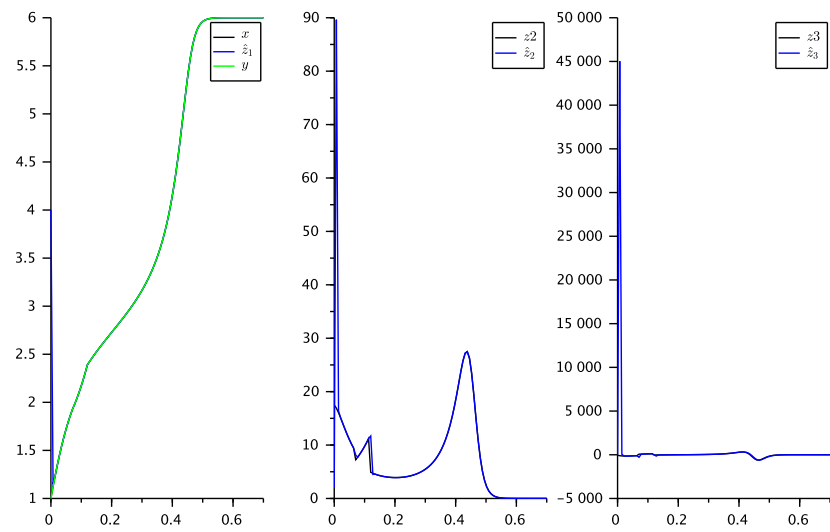

Fig. 6. The estimated first two derivatives of the output.

\section{REFERENCES}

[1] A. Astolfi, R. Ortega and A. Venkatraman, A globally exponentially convergent immersion and invariance speed observer for mechanical systems with non-holonomic constraints, Automatica, 46(1), 182-189, 2010.

[2] J. Back and J. Seo, Immersion technique for nonlinear observer design, Proceedings of the American Control Conference (ACC), Anchorage (USA), 2645-2646, 2002.

[3] J. Back and J. Seo, An algorithm for system immersion into nonlinear observer form: SISO case, Automatica, 42(2), 321-328, 2006.

[4] P. Bernard, V. Andrieu and L. Praly, Expressing an Observer in Preferred Coordinates by Transforming an Injective Immersion into a Surjective Diffeomorphism, SIAM J. Control Optim., 56(3), 23272352., 2018

[5] P. Bernard, L. Praly, V. Andrieu and H. Hammouri, On the triangular canonical form for uniformly observable controlled systems, Automatica, 85, 293-300, 2017.

[6] G. Besancon and A. Ticlea, An Immersion-Based Observer Design for Rank-Observable Nonlinear Systems, IEEE Transactions on Automatic Control, 52, 83-88, 2007.

[7] D. Dochain and A. Rapaport, An asymptotic observer for batch processes with single biogas measurement, IFAC Conference on Mod-
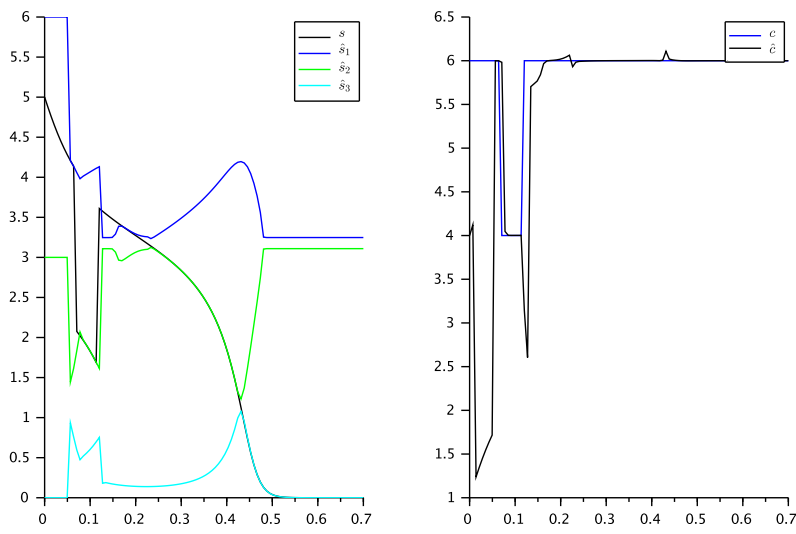

Fig. 7. Left: the estimated solutions of equation (14), with piecewise constant parameter $c$ in the case of estimated output's derivatives. Right: the constructed parameter $\hat{c}$ superposed with the exact parameter $c$.

elling, Identification and Control of Nonlinear Systems (MICNON), Guadalajara, IFAC-PapersOnLine, 51(13), 420-424, 2018.

[8] J.P. Gauthier, H. Hammouri and I. Kupka, Observers for nonlinear systems, IEEE Conference on Decision and Control, Brighton, England, 1991.

[9] J.P. Gauthier and I. Kupka, Deterministic Observation Theory and Applications. Cambridge University Press, 2001.

[10] I. Haidar, J. Barbot, A. Rapaport and M. Ghanes, Observability singularity of batch bioreactors: A solution based on high order sliding mode differentiator approach, 11th Asian Control Conference (ASCC), Gold Coast, QLD, 2017, pp. 336-338, 2017.

[11] J. Levine and R. Marino, Nonlinear system immersion, observers and finite-dimensional filters, Systems \& Control Letters, 7(2), 133-142, 1986.

[12] A. Rapaport and M. Maloum, Design of exponential observers for nonlinear systems by embedding, International Journal of Robust and Nonlinear Control, vol. 14, pp. 273-288, 2004.

[13] A. Ticlea and G. Besancon, Immersion-Based Observer Design, In: Nonlinear Observers and Applications, Lecture Notes in Control and Information Sciences 363, (Ed. G. Besancon), Springer, 2007.

[14] X. Xia and M. Zeitz, On nonlinear continuous observers, International Journal of Control, 66:6, 943-954, 1997. 